\title{
Hypersensitivity pneumonitis due to Aspergillus fumigatus in compost
}

\author{
W VINCKEN, P ROELS \\ From the Departments of Internal Medicine, Respiratory Division, Academic Hospital, University of Brussels
}

We present what may be the first reported case of acute hypersensitivity pneumonitis (allergic alveolitis) due to Aspergillus fumigatus in vegetable compost.

\section{Case report}

A previously healthy 20 year old man presented with a three day history of dyspnoea, dry cough, and fever. As his first job, he had started working two months before in a vegetable compost plant, where he had been repeatedly exposed to dust while turning the fermentation heaps with a garden fork. He was breathing shallowly and had fine crackles at both lung bases. A chest radiograph (fig 1) showed diffuse bilateral reticulonodular shadowing. His arterial oxygen tension $\left(\mathrm{PaO}_{2}\right)$ while breathing room air was $58 \mathrm{~mm} \mathrm{Hg}(7.7 \mathrm{kPa})$, arterial carbon dioxide tension $\left(\mathrm{PaCO}_{2}\right) 34 \mathrm{~mm} \mathrm{Hg}(4 \cdot 5 \mathrm{kPa})$, and $\mathrm{pH} 7 \cdot 42$. The white blood cell count was $12.9 \times 10^{9} / 1$ ( $85 \%$ neutrophils). Spirometry showed a severe restrictive deficit: forced vital capacity 1.371 (predicted 5.91); FEV 1.371 (predicted 4.9 1). Transfer coefficient (KCO) was reduced at $3.3 \mathrm{ml}$ $\mathrm{min}^{-1} \mathrm{~mm} \mathrm{Hg}^{-1}\left(1.1\right.$ (predicted 1.6) $\mathrm{mmol} \mathrm{min}^{-1}$ $\left.\mathrm{kPa}^{-1} \mathrm{l}^{-1}\right)$. The patient improved rapidly after his admission to hospital. On discharge from hospital, ten days after admission, the chest radiograph was normal.

Three weeks later, six hours after a social visit from his work mates in work clothes, the symptoms and signs he had had at his first admission recurred and he was readmitted to hospital. The chest radiograph again showed diffuse, bilateral, reticulonodular shadowing with acinar consolidation in the perihilar regions. $\mathrm{His} \mathrm{PaO}_{2}$ while breathing air was $52 \mathrm{~mm} \mathrm{Hg}$ (6.9 kPa), the $\mathrm{PaCO}_{2} 32 \mathrm{~mm} \mathrm{Hg}(4.3 \mathrm{kPa})$, and $\mathrm{pH} 7 \cdot 46$. A test for serum precipitins against $A$ fumigatus was strongly positive, while precipitins against thermophilic actinomycetes, Micropolyspora faeni, and other fungi were absent. He was given oral prednisolone $40 \mathrm{mg} / \mathrm{d}$, and recovered over 10 days, with almost complete clearing of the chest radiograph. Fourteen days after admission, however, he developed purulent sputum and haemoptysis. High fever, dyspnoea, and respiratory failure recurred. The $\mathrm{PaO}_{2}$ while he was breathing oxygen with a flow of $6 \mathrm{l} / \mathrm{min}$ via nasal prongs was $48 \mathrm{~mm} \mathrm{Hg}(6.4 \mathrm{kPa})$, $\mathrm{PaCO}_{2} 31 \mathrm{~mm} \mathrm{Hg}(4 \cdot 1 \mathrm{kPa})$, and $\mathrm{pH}$ 7.49. A chest radiograph (fig 2) showed airspace consolidation with air bronchograms of both upper lobes, the lingula, and the

Address for reprint requests: Dr W Vincken, Jan Palfijnlaan 6/3, B-1020 Brussels, Belgium

Accepted 27 June 1983

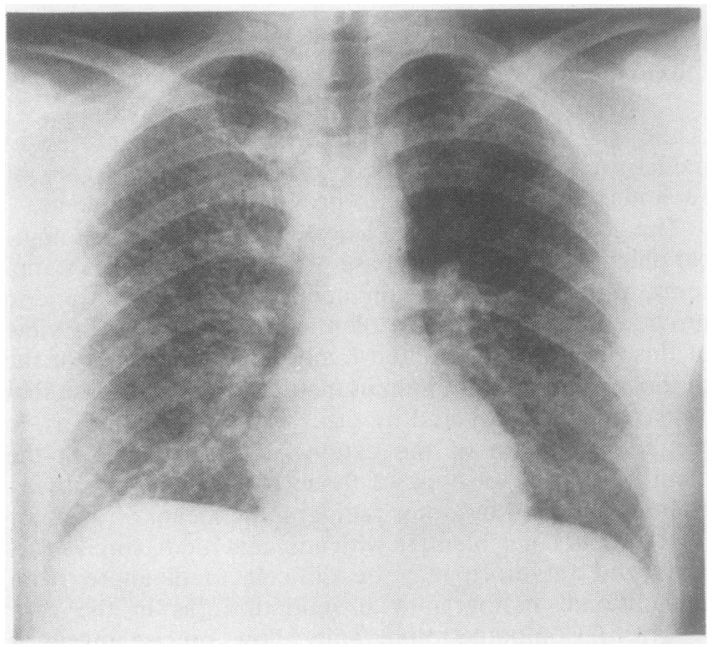

Fig 1 Posteroanterior chest radiograph at the patient's first admission for hypersensitivity pneumonitis, showing diffuse bilateral reticulonodular shadowing.

right middle lobe. Smears and cultures of sputum, blood, and urine were negative for bacteria, but bronchial washings obtained by fibreoptic bronchoscopy and multiple sputum specimens grew $A$ fumigatus. The patient was treated with amphotericin in a total daily dose of $30 \mathrm{mg}$ intravenously and $10 \mathrm{mg}$ by aerosol and with oral flucytosine $(150 \mathrm{mg} / \mathrm{kg}$ daily). Rapid reversal of the fulminant clinical course followed and arterial blood gas tensions returned to normal. Sputum cultures were all negative for $\boldsymbol{A}$ fumigatus after initiation of treatment. The chest radiograph cleared gradually over six weeks until only a few fibrotic streaks were left. Plasma protein, immunoelectrophoresis, lymphocyte and subpopulations counts, lymphoblast transformation tests (phytohaemagglutinin, concanavalin A, pokeweek mitogen), and granulocyte count and function (nitrazolium blue test) were all normal, even though the patient was still receiving prednisolone. When he was discharged from hospital amphotericin was discontinued, after a total cumulative dose of $1380 \mathrm{mg}(1060 \mathrm{mg}$ intravenously and $320 \mathrm{mg}$ by aerosol). Prednisolone was gradually withdrawn and flucytosine treatment maintained for another four weeks. A fumigatus grew abundantly on cultures of compost from the patient's previous work site.

Four months after the first admission the forced vital 


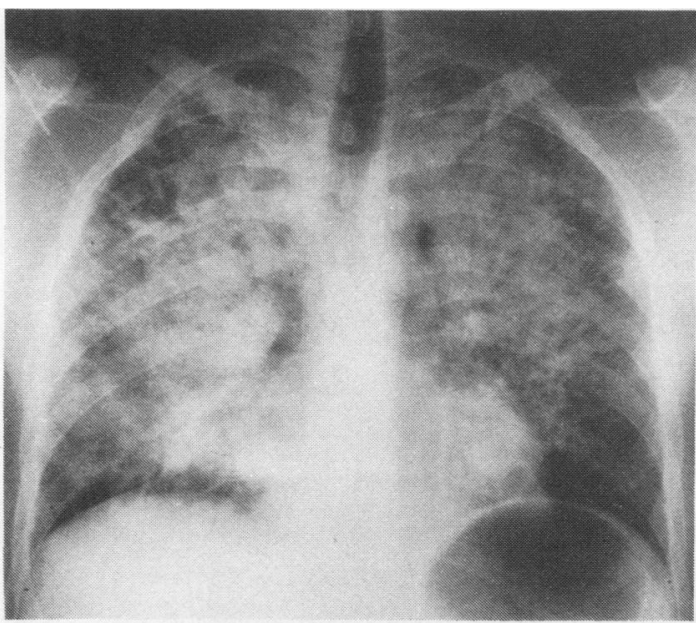

Fig 2 Posteroanterior chest radiograph after the patient had developed invasive bronchopulmonary aspergillosis complicating hypersensitivity pneumonitis, with airspace consolidation.

capacity was $3.35 \mathrm{l}, \mathrm{FEV}_{1} 2.9 \mathrm{l}$, and $\mathrm{KCO} 4 \mathrm{ml} \mathrm{min}-1$ $\mathrm{mm} \mathrm{Hg}^{-1}\left(1.3 \mathrm{mmol} \mathrm{min}^{-1} \mathrm{kPa}^{-1} \mathrm{l}^{-1}\right)$. The patient changed occupation and remains well two years later.

\section{Discussion}

The diagnosis of acute hypersensitivity pneumonitis was suspected because of the typical clinical course, the radiological features, and the occupational history of repeated heavy exposure to compost dust. Support for the diagnosis was provided by the relapse of hypersensitivity pneumonitis occurring within six hours of exposure to his work mates' work clothes. Although not a very intense re-exposure, this can certainly be regarded as equivalent to an (unintended) provocation test. The rather violent response may indicate the considerable degree of hypersensitivity, reflected in the rapid development of high levels of specific serum precipitins against $\boldsymbol{A}$ fumigatus.

Although Mullins et $\mathrm{l}^{\prime}$ have shown that vegetable compost produces large numbers of $\boldsymbol{A}$ fumigatus spores, which are liberated into the air in high concentrations if the compost heaps are disturbed, no case of hypersensitivity pneumonitis due to $A$ fumigatus from compost has been reported previously. Mushroom pickers who handle compost develop precipitins against thermophilic actinomycetes $^{23}$ and $M$ faeni, ${ }^{3}$ but not against $A$ fumigatus. Hypersensitivity pneumonitis occurring in farm workers who handle mouldy materials is almost invariably due to thermophilic actinomycetes, $M$ faeni, or both. Indeed, except for malt worker's lung, which is due to $A$ clavatus, hypersensitivity pneumonitis induced by aspergillus species is extremely rare, and in none of the reported cases has compost been handled. We believe that our patient's course may have been complicated by invasive bronchopulmonary aspergillosis. This diagnosis is supported by the following features: (1) The dramatic clinical picture developed acutely in an almost completely recovered patient. (2) Multiple cultures of bronchoscopic washings and all sputum specimens obtained after (but not before) his deterioration yielded $A$ fumigatus; in a suggestive clinical setting this has been regarded as evidence for invasive bronchopulmonary aspergillosis rather than laboratory contamination or simple colonisation of the respiratory tract. $^{4}$ (3) The rapid clinical response to a specific antifungal drug regimen.

A combination of amphotericin and flucytosine treatment has been proposed as the regimen of choice, ${ }^{5}$ allowing the total dose of amphotericin to be reduced to less toxic levels. Since in our patient there was no evidence of systemic dissemination, we gave part of the amphotericin as an aerosol in an attempt to avoid high serum concentrations while enhancing the drug concentration in the respiratory tract. The patient's rapid improvement made invasive diagnostic procedures no longer justifiable.

With rare exceptions ${ }^{4}$, invasive bronchopulmonary aspergillosis occurs in patients with an underlying immunodeficiency. ${ }^{67}$ Even though he had been receiving prednisolone in moderate doses for 10 days, our patient was not immunodeficient as judged by his responses to normal immunological tests. Conceivably the changes in the lung parenchyma due to hypersensitivity pneumonitis altered local immune defences, predisposing to invasive bronchopulmonary aspergillosis. Prompt institution of specific antifungal treatment ${ }^{8}$ and the absence of underlying immunodeficiency may explain the favourable outcome in our patient.

We wish to express our thanks to Mr Edwin Adriaenssens for his technical assistance.

\section{References}

' Mullins J, Harvey R, Seaton A. Sources and incidence of airborne Aspergillus fumigatus. Clin Allergy 1976;6:209-17.

${ }^{2}$ Bringhurst LS, Byrne RN, Gershon-Cohen J. Respiratory disease of mushroom workers: farmer's lung. JAMA 1959;171: $15-8$.

${ }^{3}$ Sakula A. Mushroom-worker's lung. Br Med J 1967;iii:708-10.

${ }^{4}$ Kennedy WPU, Malone DN, Blyth W. Necrotizing pulmonary aspergillosis. Thorax 1970;25:691-701.

5 Park GR. Drummond GB, Lamb D, et al. Disseminated aspergillosis occurring in patients with respiratory, renal and hepatic failure. Lancet 1982;ii: 179-83.

- Williams DM, Krick JA, Remington JS. Pulmonary infection in the compromized host (State of the art, Part I). Am Rev Respir Dis 1976;114:359-94.

' Pennington JE. Aspergillus lung disease. Med Clin North Am 1980;64:475-90.

${ }^{8}$ Aisner J, Schimpff S, Wiernik PH. Treatment of invasive aspergillosis: relation of early diagnosis and treatment to response. Ann Intern Med 1977;86:539-43. 